\title{
All Well-Posed Problems have Uniformly Stable and Convergent Discretizations
}

\author{
Robert Schaback \\ Univ. Göttingen \\ schaback@math.uni-goettingen.de \\ http://num.math.uni-goettingen.de/schaback/research/group.html
}

Draft of August 2, 2021

\begin{abstract}
This paper considers a large class of linear operator equations, including linear boundary value problems for partial differential equations, and treats them as linear recovery problems for objects from their data. Well-posedness of the problem means that this recovery is continuous. Discretization recovers restricted trial objects from restricted test data, and it is well-posed or stable, if this restricted recovery is continuous. After defining a general framework for these notions, this paper proves that all wellposed linear problems have stable and refinable computational discretizations with a stability that is determined by the well-posedness of the problem and independent of the computational discretization. The solutions of discretized problems converge when enlarging the trial spaces, and the convergence rate is determined by how well the full data of the object solving the full problem can be approximated by the full data of the trial objects. This allows very simple proofs of convergence rates for generalized finite elements, symmetric and unsymmetric Kansa-type collocation, and other meshfree methods like Meshless Local Petrov-Galerkin techniques. It is also shown that for a fixed trial space, weak formulations have a slightly better convergence rate than strong formulations, but at the expense of numerical integration. Since convergence rates are reduced to those coming from Approximation Theory, and since trial spaces are arbitrary, this also covers various spectral and pseudospectral methods. All of this is illustrated by examples.
\end{abstract}

\section{Introduction and Summary}

This paper focuses on mathematical problems that have solutions $u$ in some normed linear space $U$ over $\mathbb{R}$ satisfying infinitely many linear conditions that we write as

$$
\lambda(u)=f_{\lambda} \text { for all } \lambda \in \Lambda \subseteq U^{*}
$$

with given real numbers $f_{\lambda}$ and continuous linear functionals $\lambda$ on $U$ collected into a set $\Lambda \subset U^{*}$. We call the real numbers $\left\{f_{\lambda}\right\}_{\lambda \in \Lambda}$ the data that hopefully allow to identify the object $u$, which will in many cases be a multivariate function on some domain. Solving (1.1) for $u$ from given data $\left\{f_{\lambda}\right\}_{\lambda \in \Lambda}$ is a recovery problem, and we view it as posed in an abstract mathematical setting that is not directly accessible for computation. It can be called an analytical problem in contrast to the computational problems that will follow later. The transition from an analytical problem to a computational problem will be called discretization.

Typical special cases arise when solving partial differential equations (PDEs). The object $u$ to be recovered is always an element of some space $U$ of real-valued functions 
on a domain $\Omega$, but weak and strong formulations of PDEs use very different types of data, namely either integrals against test functions or derivative values at evaluation points, plus boundary conditions of various forms. If two problems use different data to identify the same object, we consider them as different here.

The PDE applications of (1.1) suggest to view the application of the functionals $\lambda \in \Lambda$ as testing a trial object $u$. Discretization will then fix a finite-dimensional trial subspace $U_{M} \subset U$ and a finite set $\Lambda_{N}$ of test functionals from $\Lambda$. We pursue this distinction between the trial and the test side of 1.1 throughout this paper.

If a problem in Mathematical Analysis is well-posed, it should have a discretization in Numerical Analysis that is also well-posed. This requires to derive some sort of numerical stability of well-chosen discretizations from the well-posedness property of the underlying analytical problem. This paper proves the above statement under mild additional assumptions after stating clearly in Sections 2 and 4 what is to be understood by well-posedness of a problem and its discretization. It turns out in Section 5 that one can choose refinable discretizations that have stability properties depending only on the well-posedness of the given problem, not on the discretizations chosen. This depends crucially on what we call a monotone refinable dense (MRD) discretization strategy in Section 4 In an older discretization theory [24, 25, 7], error bounds and convergence results depended on stability inequalities that needed complicated proofs [21], while this paper shows that one can always enforce uniform stability by sufficiently thorough testing.

The resulting discretized linear problems will be overdetermined due to this stabilization, and should be solved approximately by minimizing residuals. Section 7 deals with this, and shows that the final error bounds and convergence rates are determined by how well the data of the true solution can be approximated by the data of elements of the trial space. We call this Trial Space Data Approximation. In particular, error bounds and convergence rates are independent of the details of testing.

Section 8 extends the previous results to ill-posed problems and noisy data, while Sections 9 and 10 specialize to recovery in Hilbert spaces, where uniformly stable and sometimes optimal discretizations are readily available. These generalize finite elements and symmetric collocation, as will be explained in Section 13.2 when it comes to examples.

Stability can be spoiled by bad bases. Therefore this paper ignores bases and focuses on spaces instead, up to Section 11 where the influence of bases on the trial and the test side is studied. A very common class of bases are the nodal bases used in classical piecewise linear finite elements and various meshless methods. Many application papers report good stability properties of these, and Section 12 provides a fairly general mathematical proof, showing that convergence in the nodes can be derived from convergence of the Trial Space Data Approximation.

The paper closes with a number of examples that apply the above theory. Polynomial interpolation in Section 13.1 illustrates that the stabilization results of this paper 
imply quite some overtesting, i.e. oversampling on the test side to guarantee uniform stability. Furthermore, it points out how spectral methods are covered and why weak formulations yield slightly faster convergence than strong formulations, though for weaker norms and at the expense of numerical integration.

Section 13.2 deals with the standard setting for finite elements for homogeneous boundary conditions, showing that it fits perfectly into the framework, including extension to other trial spaces and a Petrov-Galerkin treatment.

The remaining examples address the standard Poisson problem with Dirichlet boundary conditions, for simplicity. Section 13.3 focuses on collocation as a typical strong problem. This covers various kinds of meshless methods, including Kansa's unsymmetric collocation, and it is shown how to derive specific convergence rates depending on the trial spaces chosen. The weak Dirichlet case is handled in Section 13.4 and a comparison of convergence rates for the strong and weak formulations, using the same trial spaces, is provided in Section 13.5 .

Finally, Atluri's Meshless Local Petrov Galerkin (MLPG) scheme [4] is treated in Section 13.6 This includes error bounds and convergence rates for different variations of the method, but it was necessary to include a first proof of well-posedness of the local weak form behind MLPG.

Summarizing, this paper shows that under mild hypotheses

1. all well-posed problems have uniformly stable discretizations made possible by sufficiently extensive testing, and

2. convergence rates for such discretizations can be played back to known convergence rates of Trial Space Data Approximation, i.e. the approximation of the data of the true solution by the data of the trial elements. These rates depend on what "data" means and are taken in the norm arising in the well-posedness condition.

3. Weak and strong formulations of a given background problem will have different definitions of "data" and will need different versions of well-posedness, and these differences enter into the previous item and influence the convergence rates, even when trial spaces are the same for both formulations.

4. For a given fixed trial space, it is shown that in standard applications the weak formulation converges slightly faster than the strong formulation.

5. Nodal bases have a stability advantage over other bases.

On the downside, the test strategies guaranteeing uniform stability are only shown to exist, they are not constructed. Future work needs explicit sufficient conditions on specific test strategies to guarantee uniform stability. This can be done by greedy testing as touched in earlier papers on adaptivity [28, 14, 19, 26]. Finally, emphasis so far is only on errors, convergence rates, and stability of algorithms, but not on computational 
efficiency. It is a major challenge to relate the achievable convergence rates and stability properties to computational efficiency. Anyway, this paper provides a starting point towards these goals.

\section{Problems, Data, and Well-Posedness}

Behind (1.1) there is a linear data map $D: U \mapsto \mathbb{R}^{\Lambda}=: V$ that takes each $u \in U$ into the set of values $\{\lambda(u)\}_{\lambda \in \Lambda}$ in the data space $V$. This allows to rewrite (1.1) as an operator equation

$$
D(u)=f
$$

for some given $f$ in the data space $V$. Each operator equation can be formally interpreted this way, e.g. by defining $\Lambda$ as the set of all functionals $\mu \circ D$ when $\mu$ varies in the unit sphere of $V^{*}$.

Example 2.2. As an illustration, consider the standard Dirichlet problem

$$
\begin{array}{rll}
-\Delta u & =f & \text { in } \Omega \subset \mathbb{R}^{d} \\
u=g & \text { in } \Gamma:=\partial \Omega
\end{array}
$$

where $f$ and $g$ are given functions on $\Omega$ and $\Gamma$. A strong formulation writes it in the form 1.1 with functionals

$$
\begin{array}{lll}
\lambda_{x}: & : \quad u \mapsto-\Delta u(x), & x \in \Omega \\
\mu_{y}: & u \mapsto u(y), & y \in \Gamma
\end{array}
$$

on some space $U$ where both types of functionals are continuous. The standard FEM algorithms use weak functionals

$$
\lambda_{v}: u \mapsto(\nabla u, \nabla v)_{L_{2}(\Omega)} \text { for all } v \in H_{0}^{1}(\Omega)
$$

and add the functionals $\mu_{y}$ for points on the boundary. We postpone further details to sections 13.3 and 13.4 but remark that the data maps differ considerably.

We give the data space a norm structure by requiring that

$$
\|D u\|_{V}:=\sup _{\lambda \in \Lambda}|\lambda(u)| \text { for all } u \in U
$$

is a norm on $D(U)$ that we assume to be extended to $V$, if not $V=D(U)$. We shall call this the data norm, and note that it leads to a seminorm

$$
\|u\|_{D}:=\|D u\|_{V}=\sup _{\lambda \in \Lambda}|\lambda(u)| \text { for all } u \in U
$$

on the object space $U$. This is well-defined if all functionals in $\Lambda$ are uniformly bounded. We assume existence of the data norm from now on, but remind the reader that renormalization of functionals changes the data norm and all issues depending on it, like the well-posedness conditions that we introduce later. 
Definition 2.5. An analytic problem in the sense of this paper consists of

1. a linear normed object space $U$,

2. a set $\Lambda$ of linear functionals on $U$ leading to a data map $D$ as in (2.1)

3. with values in a normed data space $V$ such that

4. (2.3) holds and is a norm on $V$.

Unique solvability of the problem (1.1) or 2.1) requires that $u \in U$ vanishes if all data $\lambda(u)$ for all $\lambda \in \Lambda$ vanish, or that $D$ is injective, or that $\|\cdot\|_{D}$ is a norm. A somewhat stronger and quantitative notion is well-posedness:

Definition 2.6. An analytic problem in the sense of Definition 2.5 is well-posed with respect to a well-posedness norm $\|\cdot\|_{W P}$ on $U$ if there is a constant $C$ such that a well-posedness inequality

$$
\|u\|_{W P} \leq C\|D u\|_{V}=C\|u\|_{D} \text { for all } u \in U
$$

holds.

This means that $D^{-1}$ is continuous as a map $D(U) \rightarrow U$ in the norms $\|\cdot\|_{V}$ and $\|\cdot\|_{W P \text {. }}$ The well-posedness norm $\|\cdot\|_{W P}$ on $U$ will often be weaker than the norm $\|\cdot\|_{U}$ on $U$ needed to let the data be well-defined.

In the context of Example 2.2 the strong problem leads to well-posedness with $\|\cdot\|_{W P}=$ $\|\cdot\|_{\infty, \Omega}$, while the weak form has $\|\cdot\|_{W P}=\|\cdot\|_{L_{2}(\Omega)}$. Details will follow in 13.3 and 13.4. but we remark here that deriving computationally useful well-posedness inequalities is a serious issue that is not satisfactorily addressed by theoreticians, because they do not use computationally useful norms on the data space. For instance, the continuous dependence of solutions of elliptic problems on the boundary data is often expressed by taking Sobolev trace spaces of fractional order on the boundary, and these spaces are far from being accessible for computation. The examples will shed some light on this issue.

Future research in Applied Mathematics should target practically useful well-posedness results based on norms that are closer to computation.

\section{Trial Space Data Approximation}

We now perform the first step of discretization by choosing a finite-dimensional trial space $U_{M} \subset U$. This allows us to approximate the data $D\left(u^{*}\right) \in V$ by data $D\left(u_{M}\right)$ for all trial elements $u_{M} \in U_{M}$ in the data norm $\|.\|_{V}$, and we denote the best approximation by $u_{M}^{*}$, i.e.

$$
\left\|D u^{*}-D u_{M}^{*}\right\|_{V}=\min _{u_{M} \in U_{M}}\left\|D u^{*}-D u_{M}\right\|_{V} .
$$

We shall rely on Approximation Theory to provide upper bounds for this, and for convergence rates for $\left\|D u^{*}-D u_{M}^{*}\right\|_{V} \rightarrow 0$ if the spaces $U_{M}$ get larger and larger. These 
rates will crucially depend on the smoothness of $u^{*}$, the trial spaces $U_{M}$, and the data map $D$. For trial spaces in spectral methods, these convergence rates may be very large, and there may even be exponential convergence. We call (3.1) the Trial Space Data Approximation, but we keep in mind that the above approximation problem is computationally hazardous, because it involves infinitely many data. We can assess $u_{M}^{*}$ only in theory, not in practice.

If the problem is well-posed in the sense of Definition 2.6 the error bounds and convergence rates of the Trial Space Data Approximation immediately carry over to error bounds and convergence rates in the well-posedness norm, via

$$
\left\|u^{*}-u_{M}^{*}\right\|_{W P} \leq C\left\|D u^{*}-D u_{M}^{*}\right\|_{V},
$$

and independent of the chosen trial space. This means that Approximation Theory provides convergence rates for certain approximate solutions of certain well-posed analytic problems, but these approximate solutions are computationally inaccessible.

In the context of Example 2.2 the functions of the trial space have to approximate function values on the boundary in both the strong and the weak case. But for the strong form we have to approximate second derivatives, while the weak form only has to approximate first derivatives. Furthermore, the well-posedness norms are different. This will lead to different convergence rates in Section 13.5.

\section{MRD Discretizations}

In what follows, we shall show how to discretize the test side of an analytic problem in the sense of Definition 2.5 in such a way that a uniformly stable and finite computational strategy exists that provides approximations $\tilde{u}_{M} \in U_{M}$ with

$$
\left\|u^{*}-\tilde{u}_{M}\right\|_{W P} \leq 2\left\|u^{*}-u_{M}^{*}\right\|_{W P} \leq 2 C\left\|D u^{*}-D u_{M}^{*}\right\|_{V} .
$$

This implies that Approximation Theory provides convergence rates for certain finitely and stably computable approximate solutions of certain well-posed analytic problems. The convergence will take place in $U$ under the well-posedness norm $\|\cdot\|_{W P}$, and the convergence rate will be the convergence rate of the Trial Space Data Approximation. Our main tool will be a monotonic refinable dense (MRD) discretization of the data space $V$ that we describe now.

No matter what the data map is, the data space $V$ should allow some form of discretization for computational purposes. We model this by restriction maps

$$
R_{N}:\left\{f_{\lambda}\right\}_{\lambda \in \Lambda} \mapsto\left\{f_{\lambda}\right\}_{\lambda \in \Lambda_{N}} \in V_{N}=\mathbb{R}^{\left|\Lambda_{N}\right|}
$$

that map $V$ into finite-dimensional data spaces $V_{N}$ over $\mathbb{R}$. The discretizations use restricted data belonging to finite subsets $\Lambda_{N}$ of $\Lambda$, and these data enter practical computation. 
On the spaces $V_{N}$ we introduce the norm

$$
\left\|R_{N}\left\{f_{\lambda}\right\}_{\lambda \in \Lambda}\right\|_{V_{N}}=\left\|\left\{f_{\lambda}\right\}_{\lambda \in \Lambda_{N}}\right\|_{V_{N}}:=\max _{\lambda \in \Lambda_{N}}\left|f_{\lambda}\right|
$$

and we get the monotonicity property

$$
\left\|R_{M} v\right\|_{V_{M}} \leq\left\|R_{N} v\right\|_{V_{N}} \text { for all } v \in V \text { and all } \Lambda_{M} \subseteq \Lambda_{N} .
$$

Refinement of two discretizations defined by sets $\Lambda_{M}$ and $\Lambda_{N}$ works by taking $\Lambda_{M} \cup \Lambda_{N}$, and by the monotonicity property this will weakly increase the discrete norms. Finally, we have

$$
\|v\|_{V}:=\sup _{R_{N}, V_{N}}\left\|R_{N} v\right\|_{V_{N}} \text { for all } v \in V
$$

following from (2.3).

But there are applications where restrictions are not defined by taking all possible finite subsets of functionals. They might require background triangulations, e.g. for finite elements, and their refinement does not simply involve taking a union of two finite sets of functionals. We can generalize the above notions by ignoring functionals:

Definition 4.2. An MRD discretization of a data space $V$ consist of a set of restrictions $\left(R_{N}, V_{N}\right)$ with the properties

1. $V_{N}$ is a normed linear space with $\operatorname{dim} V_{N}<\infty$ and norm $\|\cdot\|_{V_{N}}$,

2. $R_{N}: V \rightarrow V_{N}$ is linear,

3. there is a partially defined refinement relation $\preceq$ on the restrictions such that

4. $\left(R_{M}, V_{M}\right) \preceq\left(R_{N}, V_{N}\right)$ implies $\left\|R_{M} v\right\|_{V_{M}} \leq\left\|R_{N} v\right\|_{V_{N}}$ for all $v \in V$,

5. for each two admissible restrictions $\left(R_{M}, V_{M}\right),\left(R_{N}, V_{N}\right)$ there is a restriction $\left(R_{P}, V_{P}\right)$ such that $\left(R_{M}, V_{M}\right) \preceq\left(R_{P}, V_{P}\right)$ and $\left(R_{M}, V_{M}\right) \preceq\left(R_{P}, V_{P}\right)$,

6. (4.1) is a norm, when the sup is taken over all admissible restrictions.

This axiomatic framework is open for further discussion, of course, but we assume it in what follows. We refer to the last three properties as monotonicity, refinement, and density, using the term MRD discretization for all six properties. Note that the norm arising in the density property must be the data norm that is used in the well-posedness inequality 2.7.

The discussion preceding Definition 4.2 proved

Theorem 4.3. Each analytical problem of the form (1.1) in the sense of Definition 2.5 has a MRD discretization via taking finite subsets of functionals.

For Example 2.2, it is clear that one can focus on finitely many functionals when it comes to finite computations, but it is by no means clear which and how many are to be taken to allow a uniformly stable computational method. The refinement in the FEM case is not quite standard, but will satisfy Definition 4.2 because it still uses finite subsets of functionals. 


\section{Well-Posedness of Discretized Problems}

If we use a MRD restriction $\left(R_{N}, V_{N}\right)$ on the data together with a chosen trial space $U_{M}$, we can pose the discretized problem as the linear system

$$
R_{N} D u_{M}=R_{N} D u^{*}
$$

to be solved for $u_{M} \in U_{M}$, where the computational input data are provided by the restriction $R_{N} D u^{*}$ of the data of an exact solution $u^{*}$. Such systems will usually be overdetermined.

Since the well-posedness condition (2.7) also holds on the trial space, the discretized problem 5.1] is automatically well-posed or stable in the sense

$$
\left\|u_{M}\right\|_{W P} \leq C\left(U_{M}, V_{N}\right)\left\|R_{N} D u_{M}\right\|_{V_{N}} \text { for all } u_{M} \in U_{M}
$$

if we can prove

$$
\left\|D u_{M}\right\|_{V} \leq C\left(U_{M}, V_{N}\right)\left\|R_{N} D u_{M}\right\|_{V_{N}} \text { for all } u_{M} \in U_{M}
$$

for some stability constant $C\left(U_{M}, V_{N}\right)$.

We now can state our central result, to be proven later in a somewhat more general form.

Theorem 5.3. Assume an analytic problem (2.1) with an MRD discretization. If $U_{M}$ is an arbitrary finite-dimensional subspace of $U$, there always is a restriction $\left(R_{N}, V_{N}\right)$ such that

$$
\left\|u_{M}\right\|_{D} \leq 2\left\|R_{N} D\left(u_{M}\right)\right\|_{V_{N}} \text { for all } u_{M} \in U_{M}
$$

This holds without assuming well-posedness. If the latter is assumed by (2.7), we have

$$
\left\|u_{M}\right\|_{W P} \leq 2 C\left\|R_{N} D\left(u_{M}\right)\right\|_{V_{N}} \text { for all } u_{M} \in U_{M}
$$

with the constant $C$ from (2.7).

In contrast to (5.2), the constants in (5.4) and (5.5) are independent of $U_{M}$ and $V_{M}$, proving a uniform well-posedness or stability of the discretized problem for a rather sensible choice of $V_{N}$ after an arbitrary selection of $U_{M}$. Section 13.1 will show that this uniformity may require some hidden amount of oversampling, i.e. the dimension of $V_{N}$ may be much larger than the dimension of $U_{M}$. We call this overtesting, because it occurs on the test side of the problem. Theorem 5.3 does not give any practical hints how to care for uniformly stable testing, it just proves existence. The necessary amount of overtesting to achieve uniformly stability is left open.

It is a common observation that many instabilities arise from badly chosen bases. They sometimes disappear after introduction of better bases. To identify instabilities that can be blamed to bad bases, we refrain from introducing bases as far as possible in this paper, focusing on spaces instead of bases. 


\section{Well-Posedness of Data Discretizations}

Inspection of 5.4 shows that the analytic problem and its well-posedness are not relevant for (5.4), because the actual well-posedness condition (2.7) enters only into the trivial transition from (5.4) to 5.5. In fact, everything follows already from the notion of a MRD discretization. Well-posedness is a later add-on.

Lemma 6.1. Consider a data space $V$ and associated MRD restrictions $\left(R_{N}, V_{N}\right)$ satisfying the assumptions of Section 4 Then for each finite-dimensional subspace $W_{M}$ of $V$ there always is a restriction $\left(R_{N}, V_{N}\right)$ such that

$$
\left\|w_{M}\right\|_{V} \leq 2\left\|R_{N} w_{M}\right\|_{V_{N}} \text { for all } w_{M} \in W_{M}
$$

Proof: Define $K \subset W_{M}$ as the unit sphere of $W_{M}$ defined via the norm $\|\cdot\|_{V}$. By compactness, for each $\varepsilon>0$ we can cover $K$ by finitely many $\varepsilon$-neighborhoods

$$
U_{\varepsilon}\left(y_{j}\right):=\left\{y \in K:\left\|y-y_{j}\right\|_{V} \leq \varepsilon\right\}, 1 \leq j \leq n
$$

with elements $y_{1}, \ldots, y_{n} \in K$. By the density property 4.1 we can find restrictions $R_{N_{1}}, \ldots, R_{N_{n}}$ with associated spaces $V_{N_{1}}, \ldots, V_{N_{n}}$ such that

$$
\left\|y_{j}\right\|_{V} \leq\left\|R_{N_{j}} y_{j}\right\|_{V_{N_{j}}}+\varepsilon, 1 \leq j \leq n
$$

and by repeated application of the refinement property we can define $R_{N}$ and $V_{N}$ as the "union" of these, and then

$$
\left\|R_{N_{j}} v\right\|_{V_{N_{j}}} \leq\left\|R_{N} v\right\|_{V_{N}} \text { for all } v \in V, 1 \leq j \leq n
$$

by monotonicity.

We now take an arbitrary $w_{M} \in K$ and get some $j, 1 \leq j \leq n$ with $\left\|w_{M}-y_{j}\right\|_{V} \leq \varepsilon$ via the covering. This implies $\left\|R_{N} w_{M}-R_{N} y_{j}\right\|_{V_{N}} \leq \varepsilon$ by the density property, and then

$$
\begin{aligned}
\left\|R_{N} w_{M}\right\|_{V_{N}} & \geq\left\|R_{N} y_{j}\right\|_{V_{N}}-\varepsilon \\
& \geq\left\|R_{N_{j}} y_{j}\right\|_{V_{N_{j}}}-\varepsilon \\
& \geq\left\|y_{j}\right\|_{V}-2 \varepsilon \\
& \geq\left\|w_{M}\right\|_{V}-3 \varepsilon \\
& =1-3 \varepsilon
\end{aligned}
$$

proving

$$
\left\|R_{N} w_{M}\right\|_{V_{N}} \geq(1-3 \varepsilon)\left\|w_{M}\right\|_{V}
$$

for all $w_{M} \in W_{M}$, and the assertion follows for $\varepsilon=1 / 6$.

The proof of Theorem 5.3 now follows by setting $W_{M}=D\left(U_{M}\right)$ with an arbitrary data $\operatorname{map} D$. 


\section{Solving Discretized Problems}

After choosing a trial space $U_{M}$ and getting a suitable data restriction $\left(R_{N}, V_{N}\right)$ for Theorem 5.3, the discretized recovery problem (5.1) requires computation of some $u_{M} \in U_{M}$ from the data $R_{N} D u^{*}$, where $u^{*}$ is the true solution to the analytical problem. This will usually lead to an overdetermined linear system after choosing bases, but we do not want to consider bases unless absolutely necessary.

The simplest basis-free computational method we could propose is to minimize the residual norm $\left\|R_{N} D\left(u^{*}-u_{M}\right)\right\|_{V_{N}}$ over all $u_{M} \in U_{M}$, which is a finite-dimensional approximation problem. A good candidate in the trial space $U_{M}$ is the best approximation $u_{M}^{*}$ to the solution $u^{*}$, and we are free to consider the best approximation in one of the norms $\|.\|_{U},\|.\|_{W P}$ or $\|.\|_{D}$. We do not insist here on using the data norm and a best approximation, as we did in 3.1. Instead, we keep the choice of $u_{M}^{*}$ free and are satisfied with computing an element $\tilde{u}_{M} \in U_{M}$ with

$$
\left\|R_{N} D\left(u^{*}-\tilde{u}_{M}\right)\right\|_{V_{N}} \leq C_{A}\left\|R_{N} D\left(u^{*}-u_{M}^{*}\right)\right\|_{V_{N}},
$$

however it is calculated, with a fixed constant $C_{A} \geq 1$ that makes computational life easier when chosen not too close to one. We call $u_{M}^{*}$ a comparison trial object. It is usually provided by some result of Approximation Theory that yields a useful bound on the right-hand side of 7.1 . Due to the monotonicity and density properties, we also have

$$
\left\|R_{N} D\left(u^{*}-\tilde{u}_{M}\right)\right\|_{V_{N}} \leq C_{A}\left\|R_{N} D\left(u^{*}-u_{M}^{*}\right)\right\|_{V_{N}} \leq C_{A}\left\|D\left(u^{*}-u_{M}^{*}\right)\right\|_{V},
$$

such that the best Trial Space Data Approximation is always an upper bound.

Anyway, 7.1 implies

$$
\begin{aligned}
\left\|\tilde{u}_{M}-u_{M}^{*}\right\|_{D} & \leq 2\left\|R_{N} D\left(\tilde{u}_{M}-u_{M}^{*}\right)\right\|_{V_{N}} \\
& \leq 2\left\|R_{N} D\left(\tilde{u}_{M}-u^{*}\right)\right\|_{V_{N}}+2\left\|R_{N} D\left(u^{*}-u_{M}^{*}\right)\right\|_{V_{N}} \\
& \leq\left(2 C_{A}+2\right)\left\|R_{N} D\left(u^{*}-u_{M}^{*}\right)\right\|_{V_{N}} \\
& \leq\left(2 C_{A}+2\right)\left\|D\left(u^{*}-u_{M}^{*}\right)\right\|_{V} \\
& =\left(2 C_{A}+2\right)\left\|u^{*}-u_{M}^{*}\right\|_{D}
\end{aligned}
$$

and

$$
\begin{aligned}
\left\|\tilde{u}_{M}-u^{*}\right\|_{D} & \leq\left\|\tilde{u}_{M}-u_{M}^{*}\right\|_{D}+\left\|u_{M}^{*}-u^{*}\right\|_{D} \\
& \leq\left(2 C_{A}+3\right)\left\|u_{M}^{*}-u^{*}\right\|_{D},
\end{aligned}
$$

proving that the error of the computational solution $\tilde{u}_{M}$ is up to a factor the same as the error of the comparison trial object $u_{M}^{*}$, evaluated in the data norm.

Theorem 7.2. Assume an MRD discretization of an analytic problem along the lines of the previous sections. Then each computational technique to solve the discretized problem approximatively by an element $\tilde{u}_{M} \in U_{M}$ such that (7.1) holds, will also guarantee

$$
\left\|\tilde{u}_{M}-u^{*}\right\|_{D} \leq\left(2 C_{A}+3\right)\|\| u_{M}^{*}-u^{*} \|_{D}
$$

for any comparison trial object $u_{M}^{*}$. 
Corollary 7.3. Adding well-posedness to Theorem 7.2 yields

$$
\left\|u^{*}-\tilde{u}_{M}\right\|_{W P} \leq C\left(2 C_{A}+3\right)\left\|u_{M}^{*}-u^{*}\right\|_{D}
$$

proving that convergence rates in the data norm transfer to the same convergence rates in the norm $\|.\|_{W P}$ on $U$ arising in the well-posedness condition (2.3).

We summarize what we have so far, for easy reference in the examples.

Theorem 7.4. Assume a well-posed analytic problem with an MRD discretization as in Definitions 2.5 2.6 and 4.2 Then for arbitrary trial spaces one can choose uniformly stable test discretizations to get uniformly stable computational methods based on some form of residual minimization. The convergence rates, measured in the well-posedness norm, are given by the convergence rate of the Trial Space Data Approximation, i.e. the rate in which the data of the true solution are approximated by the data of comparison trial objects, measured in the data norm.

This will be applied in the following way. First, one assumes additional regularity of the solution object $u^{*}$ and fixes a well-known approximation process in $U$ that provides good comparison trial objects $u_{M}^{*}$ for these trial spaces, and with a very good convergence rate $u_{M}^{*} \rightarrow u^{*}$ that may even be spectral in a weak norm like $\|\cdot\|_{W P}$. Then these approximations are used for comparison in the above theory, and the convergence rate in the data norm is calculated from what is known about the approximation process. Then we know that this rate is the one that arises when solving the analytic problem, and it arises in the well-posedness norm. This may even yield spectral convergence, and we shall provide examples. But note that the rate of convergence of our discretized solutions of the analytical problems is only the rate the convergence obtained after the data map is applied, and it involves the norm $\|.\|_{W P}$ used in the well-posedness condition.

The above approach applies to a large variety of well-posed analytic problems, and shows that for properly chosen scales of trial spaces $U_{M}$ and properly chosen test strategies depending on each $U_{M}$ one gets uniformly stable and convergent computational methods with convergence rates that can be derived from results of Approximation Theory. These rates normally improve with the smoothness of the true solution, but they also depend on the data map and the well-posedness norm. For a given PDE problem like in Example 2.2 the convergence rates of strong and weak formulations will be different, even if the trial spaces are the same. This is due to the fact that the data maps, data norms, and well-posedness norms are different. Details will follow in Section 13.5

If the true solution necessarily has certain singularities of a known type, like in elliptic PDE problems on domains with incoming corners, one should always add the correct singular functions to the trial space. Then the approximation quality of the singular solution in the augmented trial space is the same as the approximation quality of a regular solution in the original trial space, and this quality will improve with the smoothness of the regular solution. In this sense, going over to extended trial spaces like in the XFEM or GFEM does not need a new theory here. 


\section{Noisy Data}

Corollary 7.3 showed that ill-posed problems can be treated, if one is satisfied with reproducing the data well. But so far we always have assumed that the input data are given exactly as data of an existing solution. If this is not true, a few changes are necessary. We assume that the data map $D$ is always exact, but the input data for computations are assumed to be polluted by either noise or errors in evaluating the data functionals. This also handles the error committed by numerical integration when the data functionals of weak PDE problems are considered.

The data now consist of a general element $v^{*}$ of the data space $V$, and we assume that there is an object $u^{*} \in U$ such that $\left\|D\left(u^{*}\right)-v^{*}\right\|_{V}$ is small, and we want to recover this object well, or others with a similarly good data reproduction. We choose a trial space $U_{M} \subset U$ as before, and we define $W_{M}:=D\left(U_{M}\right) \subset V$ as at the end of Section 6. Clearly, (7.1) now has to be replaced by

$$
\left\|R_{N} v^{*}-R_{N} D \tilde{u}_{M}\right\|_{V_{N}} \leq C_{A}\left\|R_{N} v^{*}-R_{N} D u_{M}^{*}\right\|_{V_{N}},
$$

because there are no other data at hand. Then

Theorem 8.2. Assume an MRD discretization of an analytic problem along the lines of the previous sections. Then each computational technique to solve the discretized problem approximatively by an element $\tilde{u}_{M} \in U_{M}$ such that (8.1) holds, will guarantee

$$
\left\|\tilde{u}_{M}-u^{*}\right\|_{D} \leq\left(2 C_{A}+3\right)\|\| u_{M}^{*}-u^{*}\left\|_{D}+\left(2 C_{A}+2\right)\right\| v^{*}-D u^{*} \|_{V}
$$

for any $u^{*} \in U$.

Proof: We proceed like above, via

$$
\begin{aligned}
\left\|\tilde{u}_{M}-u_{M}^{*}\right\|_{D} & \leq 2\left\|R_{N} D\left(\tilde{u}_{M}-u_{M}^{*}\right)\right\|_{V_{N}} \\
& \leq 2\left\|R_{N} D \tilde{u}_{M}-R_{N} v^{*}\right\|_{V_{N}}+2\left\|R_{N} v^{*}-R_{N} D u_{M}^{*}\right\|_{V_{N}} \\
& \left.\leq\left(2 C_{A}+2\right) \| R_{N} v^{*}-R_{N} D u_{M}^{*}\right) \|_{V_{N}} \\
& \leq\left(2 C_{A}+2\right)\left(\left\|R_{N} v^{*}-R_{N} D u^{*}\right\|_{V_{N}}+\left\|R_{N} D u^{*}-R_{N} D u_{M}^{*}\right\|_{V_{N}}\right) \\
& \left.\leq\left(2 C_{A}+2\right)\left(\| v^{*}-D u^{*}\right)\left\|_{V}+\right\| D\left(u^{*}-u_{M}^{*}\right) \|_{V}\right) \\
& \left.\leq\left(2 C_{A}+2\right)\left(\| v^{*}-D u^{*}\right)\left\|_{V}+\right\| u^{*}-u_{M}^{*} \|_{D}\right)
\end{aligned}
$$

and get

$$
\begin{aligned}
\left\|\tilde{u}_{M}-u^{*}\right\|_{D} & \leq\left\|\tilde{u}_{M}-u_{M}^{*}\right\|_{D}+\left\|u_{M}^{*}-u^{*}\right\|_{D} \\
& \leq\left(2 C_{A}+3\right)\left\|u_{M}^{*}-u^{*}\right\|_{D}+\left(2 C_{A}+2\right)\left\|v^{*}-D u^{*}\right\|_{V} .
\end{aligned}
$$

The inequality (8.3) shows that errors in the data functionals, e.g. integration errors for weak data, can spoil the convergence unless they are at least as small as the error committed by the comparison object $u_{M}^{*}$ in the data norm. For trial spaces that allow fast convergence, the admissible errors in the data functionals are severely restricted by this observation. 
One can go into 8.1) by choosing $u_{M}^{*}$ as the minimizer of $\left\|v^{*}-D\left(u_{M}\right)\right\|_{V}$ over all $u_{M} \in U_{M}$. Then

$$
\left\|R_{N} v^{*}-R_{N} D\left(\tilde{u}_{M}\right)\right\|_{V_{N}} \leq C_{A}\left\|R_{N} v^{*}-R_{N} D\left(u_{M}^{*}\right)\right\|_{V_{N}} \leq C_{A}\left\|v^{*}-D u_{M}^{*}\right\|_{V},
$$

and the proof of Theorem 8.2 yields

$$
\begin{aligned}
\left\|\tilde{u}_{M}-u_{M}^{*}\right\|_{D} & \left.\leq\left(2 C_{A}+2\right) \| R_{N} v^{*}-R_{N} D u_{M}^{*}\right) \|_{V_{N}} \\
& \left.\leq\left(2 C_{A}+2\right) \| v^{*}-D u_{M}^{*}\right) \|_{V} \\
\left\|v^{*}-D \tilde{u}_{M}\right\|_{D} & \leq\left\|v^{*}-D u_{M}^{*}\right\|_{D}+\left\|D u_{M}^{*}-D \tilde{u}_{M}\right\|_{D} \\
& \leq\left(2 C_{A}+3\right)\left\|v^{*}-D u_{M}^{*}\right\|_{D} .
\end{aligned}
$$

Corollary 8.4. Assume an analytic problem that has a MRD discretization without being well-posed, and assume that the given data do not necessarily come from some solution $u^{*}$. Then there is a uniformly stable computational strategy that provides trial elements that reproduce the given data at the quality of the Trial Space Data Approximation. This reduces the error and convergence analysis to an approximation problem for a data element $v^{*} \in V$ by a data subspace $D\left(U_{M}\right)$ in $V$ under the norm in $V$.

If there is no well-posedness, there still is a backward error analysis. Instead of solving the problem with data $v^{*}$, which may be unsolvable or ill-posed, one can come up with an element $\tilde{u}_{M}$ from the trial space which has data that are close to the given data, and roughly as close as possible for the given trial space. For PDE solving, this usually means that one has an exact solution of a PDE with perturbed boundary data and a perturbation in the inhomogeneity of the PDE. If these perturbations are calculated and turn out to be tolerable, the user might be satisfied with $\tilde{u}_{M}$. Many application papers proceed this way, unfortunately, but users should always keep in mind that there may be very different trial elements that reproduce the data nicely, if there is no wellposedness.

\section{Discretization in Hilbert Spaces}

We now assume that $U$ is a Hilbert space with inner product $(., .)_{U}$ and that the data map is composed of continuous functionals $\lambda \in \Lambda \subset U^{*}$ like in the beginning of section 4 . The Riesz map allows a transition from functionals to functions, and thus we can fix a finite subset $\Lambda_{N}=\left\{\lambda_{1}, \ldots, \lambda_{N}\right\} \subset \Lambda$ and consider the Riesz representers $u_{1}, \ldots, u_{N} \in U$ of these functionals. If linear independence is assumed, we have $N$-dimensional spaces $L_{N} \subset U^{*}$ and $U_{N} \subset U$ by taking the spans, and the space $V_{N}$ is $\mathbb{R}^{N}$ as the range of the restriction $R_{N}$ with $R_{N} D u=\left(\lambda_{1}(u), \ldots, \lambda_{N}(u)\right)^{T}$ which just is the usual projection from $V:=\mathbb{R}^{\Lambda}$ to $V_{N}:=\mathbb{R}^{\Lambda_{N}}=\mathbb{R}^{N}$. If orthonormal bases are chosen, we have the 2-norm of coefficients as $\|u\|_{U}$ for all $u \in U$, but in order to comply with Section 4, we have to take the sup-norm in the range of the data map, which is the identity if discretized in that basis. But then the identity map is not well-posed, due the choice of norms which is not adequate for Hilbert spaces. 
We thus have to change the setting, taking the norms in $V_{N}=\mathbb{R}^{N}$ as 2-norms, assuming $\Lambda$ to be countable and total, taking orthonormal bases, and the restrictions as projections focusing on finite subsets of indices in the expansions. For the choice of $U_{N}$ and $V_{N}$ as above, we then have (5.4) and (5.5) with the constant 1.

This is the standard situation in Rayleigh-Ritz-Galerkin methods. It might be surprising that everything is perfectly well-conditioned here, but this is no miracle because we focused on spaces, not on bases, and used an optimal basis for the theoretical analysis. The usual problems with conditions of stiffness matrices etc. are basis-dependent, not space-dependent.

\section{Optimal Recovery in Hilbert Spaces}

When starting from a finite set $\Lambda_{N}$ of functionals providing the data $\lambda_{j}\left(u^{*}\right)$ of a true solution of the analytic problem, the above choice of a trial space as the space spanned by the representers of the functionals is optimal under all other choices of trial spaces. This is a standard result in the theory of Reproducing Kernel Hilbert Spaces, but we include it here in a general form, because of its central importance within the context of studying all possible discretizations.

Theorem 10.1. Assume that we have a computational problem posed in a Hilbert space $U$, and the only available data are of the form $\lambda_{1}\left(u^{*}\right), \ldots, \lambda_{N}\left(u^{*}\right)$ for $N$ linearly independent data functionals in $U^{*}$ and an unknown object $u^{*} \in U$. Then, for any linear functional $\mu \in U^{*}$, consider all possible linear computational procedures for calculating good approximations of $\mu\left(u^{*}\right)$ using only the above values. Then there is a unique error-optimal strategy that works as follows:

1. Use the representers $u_{1}, \ldots, u_{N} \in U$ of the functionals $\lambda_{1}, \ldots, \lambda_{N} \in U^{*}$.

2. Calculate the interpolant $\tilde{u}$ to $u^{*}$ in the span of the representers, i.e. solve the system

$$
\lambda_{k}\left(u^{*}\right)=\sum_{j=1}^{N} c_{j} \lambda_{k}\left(u_{j}\right)=\sum_{j=1}^{N} c_{j}\left(u_{k}, u_{j}\right)_{U}=\sum_{j=1}^{N} c_{j}\left(\lambda_{k}, \lambda_{j}\right)_{U^{*}}, 1 \leq k \leq N
$$

and define

$$
\tilde{u}=\sum_{j=1}^{N} c_{j} u_{j}
$$

3. For each data functional $\mu \in U^{*}$, use the value

$$
\mu(\tilde{u})=\sum_{j=1}^{N} c_{j} \mu\left(u_{j}\right)
$$

as an approximation to $\mu\left(u^{*}\right)$. 
This approximation has minimal error under all other linear computational procedures using the same data for calculating approximations of $\mu\left(u^{*}\right)$, in the sense that the error functional has minimal norm.

This technique is independent of well-posedness and makes optimal use of the available data, error-wise. From the previous section we conclude that it is uniformly stable when considered in terms of spaces, not bases. If applied to PDE solving, it is realized by symmetric collocation [26]. It can also be applied to numerical integration and numerical differentiation, see e.g. [10, 27].

In the context of this paper, the above result shows that the quest for good trial spaces and well-posed discretizations has a simple solution in the Hilbert space situation. We shall come back to this in Section 13.5 when we look at the differences between weak and strong formulations.

\section{Bases}

We now assume that we have a well-posed analytic problem in the sense of Section 2 with an MRD discretization, and by a proper choice of restrictions $\left(R_{N}, V_{N}\right)$ according to Theorem 5.3. we have uniform stability in the form of (5.5). We specialize here to the case of Theorem 4.3 where we have functionals $\lambda \in \Lambda$ and restrictions working via subsets $\Lambda_{N} \subset \Lambda$ selecting finitely many data. We now choose a basis $u_{1}, \ldots, u_{M}$ of $U_{M}$ and take the functionals $\lambda_{1}, \ldots, \lambda_{N}$ from the set $\Lambda_{N}$. Then we consider the discretized system

$$
\sum_{j=1}^{M} a_{j} \lambda_{k}\left(u_{j}\right) \approx f_{\lambda_{k}}=\lambda_{k}\left(u^{*}\right), 1 \leq k \leq N
$$

that we solve approximatively by residual minimization like in Section 7 Clearly, a bad choice of bases will spoil stability, but we want to study this effect in detail. We quantify the stability of the object basis by norm equivalence

$$
c_{M}\left\|u_{a}\right\|_{W P} \leq\|a\|_{M} \leq C_{M}\left\|u_{a}\right\|_{W P} \text { for all } a \in \mathbb{R}^{M},
$$

with an unspecified norm $\|\cdot\|_{M}$ on $\mathbb{R}^{M}$ that is used in computation. With the $N \times M$ matrix $A=\left(\lambda_{k}\left(u_{j}\right)\right)_{1 \leq j \leq M, 1 \leq k \leq N}$. and the basis representation

$$
u_{a}:=\sum_{j=1}^{M} a_{j} u_{j}
$$

with coefficient vectors $a \in \mathbb{R}^{M}$, we see that $A a=R_{N} D u_{a}$ holds. and get

$$
\begin{aligned}
\|a\|_{M} & \leq C_{M}\left\|u_{a}\right\|_{W P} \\
& \leq C C_{M}\left\|u_{a}\right\|_{D} \\
& \leq 2 C C_{M}\left\|R_{N} D u_{a}\right\|_{V_{N}} \\
& =2 C C_{M}\|A a\|_{V_{N}}
\end{aligned}
$$

by (2.7), and (5.4). 
Theorem 11.2. Under the above assumptions, the system (11.1) has the stability property

$$
\|a\|_{M} \leq 2 C C_{M}\|A a\|_{V_{N}} \text { for all } a \in \mathbb{R}^{M} .
$$

In Section7 we minimized $\left\|R_{N}\left(D u^{*}-D u_{M}\right)\right\|_{V_{N}}$ over all $u_{M} \in U_{M}$. After introducing a basis in $U_{M}$, this is the same as minimization of $\|f-A a\|_{V_{N}}$ with $f:=R_{N} D u^{*}=$ $\left(\lambda_{1}\left(u^{*}\right), \ldots, \lambda_{N}\left(u^{*}\right)\right)^{T} \in \mathbb{R}^{N}$ over all $a \in \mathbb{R}^{M}$. We are satisfied with a vector $\tilde{a} \in \mathbb{R}^{M}$ such that

$$
\|f-A \tilde{a}\|_{V_{N}} \leq \min _{a \in \mathbb{R}^{M}}\|f-A a\|_{V_{N}} \leq C_{A}\left\|f-A a^{*}\right\|_{V_{N}},
$$

where $a^{*}$ is a good coefficient vector for the direct approximation of the true solution $u^{*}$ by elements of the trial space $U_{M}$. We use $a^{*}$ in the way we used $u_{M}^{*}$ in Section 7 as a competitor that may come from some special approximation technique. Then we form the elements $\tilde{u}_{M}:=u_{\tilde{a}}, u_{M}^{*}=u_{a^{*}} \in U_{M}$ and see that (7.1) is satisfied.

This lets us arrive at Theorem 7.2 implying that the convergence rate is the same as the rate for the Trial Space Data Approximation, but this does not yield error bounds in terms of coefficients. However, we can proceed by

$$
\begin{aligned}
\frac{1}{2 C C_{M}}\left\|a^{*}-\tilde{a}\right\|_{M} & \leq\left\|A\left(a^{*}-\tilde{a}\right)\right\|_{V_{N}} \\
& \leq\left\|A a^{*}-f\right\|_{V_{N}}+\|f-A \tilde{a}\|_{V_{N}} \\
& \leq\left(1+C_{A}\right)\left\|A a^{*}-f\right\|_{V_{N}}
\end{aligned}
$$

and get

$$
\left\|a^{*}-\tilde{a}\right\|_{M} \leq\left(1+C_{A}\right)\left(2 C C_{M}\right)\left\|A a^{*}-f\right\|_{V_{N}} .
$$

The norm in $V_{N}$ must be chosen to comply with Section 4 and this works for the discrete sup norm. But if users do not want to minimize $f-A a$ in the sup norm, an additional norm equivalence comes into play, now on $V_{N}$, and this will often depend on $\operatorname{dim} V_{N}$. In detail, norm equivalence in $V_{N}$ is assumed as

$$
c_{N}\left\|R_{N} v\right\|_{V_{N}} \leq\left\|R_{N} v\right\|_{N} \leq C_{N}\left\|R_{N} v\right\|_{V_{N}} \text { for all } v \in V .
$$

and minimization in the new norm $\|\cdot\|_{N}$ will replace (11.3) by

$$
\|f-A \tilde{a}\|_{N} \leq \min _{a \in \mathbb{R}^{M}}\|f-A a\|_{N} \leq C_{A}\left\|f-A a^{*}\right\|_{N},
$$

and our above argumentation now yields

$$
\left\|a^{*}-\tilde{a}\right\|_{M} \leq \frac{\left(1+C_{A}\right)\left(2 C C_{M}\right)}{c_{N}}\left\|A a^{*}-f\right\|_{N} .
$$

If bases are chosen badly, the quotient $C_{M} / c_{N}$ can be extremely large and will spoil the uniformity that we had so far.

Users can check their stiffness matrices $A$ computationally for stability, but Theorem 11.2 indicates that there may be a strong influence due to a bad choice of the trial basis. Even a calculation of a Singular Value Decomposition will not be completely basisindependent, since it only eliminates orthogonal basis transformations in the domain and range of $A$. 


\section{Nodal Bases}

In meshless methods, it is customary to write everything "entirely in terms of nodes" [6], which means that the functions $u_{M}$ in the trial space $U_{M}$ are parametrized by their values at certain nodes $x_{1}, \ldots, x_{M}$, i.e.

$$
u_{M}(x)=\sum_{j=1}^{M} s_{j}(x) u_{M}\left(x_{j}\right) \text { for all } u_{M} \in U_{M}
$$

with shape functions $s_{j}$ that are usually localized around $x_{j}$ and have the Lagrange property $s_{j}\left(x_{k}\right)=\delta_{j k}, 1 \leq j, k \leq M$. We prefer the term nodal basis, because there is nothing meshless in the above representation, and the standard finite elements, which nobody would call meshless, are nodal as well in the above sense. Many application papers report experimentally that these bases have favorable stability properties, and we shall now show why.

Theorem 12.1. Assume a well-posed problem in the sense of (2.7), where $U$ is a space of functions on some domain $\Omega$. Furthermore, assume that the point evaluation functionals $\delta_{x}$ are uniformly bounded by $\gamma>0$ in the norm $\|.\|_{W P}$. Finally, assume that the data space $V$ and the restrictions $V_{N}$ are normed via supremum norms, as mentioned in Section 4 and Theorem 4.3 as a special case. Then for each trial space $U_{M} \subset U$ with a nodal basis $s_{1}, \ldots, s_{M}$ using nodes $x_{1}, \ldots, x_{M} \in \Omega$ one can find a finite set of functionals $\lambda_{1}, \ldots, \lambda_{N}$ such that the $N \times M$ stiffness matrix $A$ with entries $\lambda_{j}\left(s_{k}\right)$ has the uniform stability property

$$
\|a\|_{\infty} \leq 2 \gamma C \| \text { Aa } \|_{\infty} \text { for all } a \in \mathbb{R}^{M} .
$$

Proof: We apply Theorem5.3. Then

$$
\left|u_{M}\left(x_{j}\right)\right| \leq \gamma\left\|u_{M}\right\|_{W P} \leq 2 \gamma C\left\|R_{N} D\left(u_{M}\right)\right\|_{V_{N}}=2 \gamma C \max _{\lambda_{k} \in \Lambda_{N}}\left|\sum_{j=1}^{M} \lambda_{k}\left(s_{j}\right) u_{M}\left(x_{j}\right)\right|
$$

and if we denote the vector of nodal values by $u_{X} \in \mathbb{R}^{M}$, we see that

$$
\left\|u_{X}\right\|_{\infty} \leq 2 \gamma C\left\|A u_{X}\right\|_{\infty}
$$

with the stiffness matrix $A$ with entries $\lambda_{k}\left(s_{j}\right)$.

This means that all trial spaces with nodal bases can be uniformly stabilized by taking good and large selections of test functionals. Furthermore, Section 7 provides convergence proofs and convergence rates for such techniques.

We now go closer to what user would do. In the notation of Section 11 they would invoke a least-squares solver minimizing $\|f-A a\|_{2}$ instead of minimizing $\| R_{N} D\left(u^{*}-\right.$ $\left.u_{M}\right) \|_{V_{N}}$ which is $\|f-A a\|_{\infty}$ in terms of linear algebra. In the notation of Section 11, we then have $\|\cdot\|_{M}=\|\cdot\|_{\infty, \mathbb{R}^{M}},\|\cdot\|_{N}=\|\cdot\|_{\ell_{2}, \mathbb{R}^{N}}, C_{M}=\gamma,\|\cdot\|_{V_{N}}=\|\cdot\|_{\infty, \mathbb{R}^{N}}, c_{N}=1$, leading to

$$
\left\|a^{*}-\tilde{a}\right\|_{\infty} \leq 2 \gamma C\left(1+C_{A}\right)\left\|A a^{*}-f\right\|_{2}
$$


for any reference approximation $a^{*}$. In this case, we may take $a^{*}$ as the vector of nodal values of the true solution, and then

$$
\max _{1 \leq j \leq M}\left|u^{*}\left(x_{j}\right)-\tilde{u}_{M}\left(x_{j}\right)\right| \leq 2 \gamma C\left(1+C_{A}\right) \max _{1 \leq k \leq N}\left|\lambda_{k}\left(u^{*}-s^{*}\right)\right|
$$

where $s^{*}$ is the trial function with the nodal values of the true solution.

Corollary 12.4. Assume a well-posed analytic problem with an MRD discretization, and assume that a trial space is parametrized by a nodal basis. Then the error of a computational procedure as in Section 7 evaluated on the nodes, is pointwise bounded by the error of the Trial Space Data Approximation, i.e. the approximation of the data of the true solution by the data of trial elements, measured in the data norm.

This means that using a nodal basis transfers the results of Theorem 7.4 directly to a convergence on the nodes. This is a very useful result for many meshless methods using nodal bases, e.g. when applying Moving Least Squares techniques.

\section{Examples}

\subsection{Interpolation}

For illustration, we start with the rather simple case of recovering a function $u$ on some compact domain $\Omega \subset \mathbb{R}^{d}$ from data of $u$ that do not involve derivatives. A strong formulation takes $\Lambda=\left\{\delta_{x}: x \in \Omega\right\}$ on a space $U$ on which these functionals are continuous, e.g. $U=C(\Omega)$ under the sup norm. A weak formulation uses different data, e.g. functionals

$$
\lambda_{v}(u):=(u, v)_{L_{2}(\Omega)} \text { for all } u, v \in U:=L_{2}(\Omega)
$$

and

$$
\Lambda:=\left\{\lambda_{v}:\|v\|_{L_{2}(\Omega)}=\left\|\lambda_{v}\right\|_{L_{2}^{*}(\Omega)}=1\right\} .
$$

The strong case takes $V:=C(\Omega)=U$ under the sup norm, while the weak case uses $V:=L_{2}(\Omega)=U$ under the $L_{2}$ norm. In both cases, the data map is the identity, and we have well-posedness in the norms $\|\cdot\|_{W P}=\|\cdot\|_{U}$ in both cases, but the norms differ.

The restrictions can work by selection of finitely many functionals in both cases, and all axioms of Section 4 are satisfied.

We now fix an arbitrary finite-dimensional trial space $U_{M} \subset U$, and Theorem5.3yields that there is a restriction that makes the linear system (5.1) uniformly stable in the sense (5.5) with $C=1$.

The computational procedures of Section 7 can use a comparison trial object $u_{M}^{*}$ that is the best approximation to the true solution $u^{*}$ in the norm $\|\cdot\|_{U}=\|\cdot\|_{W P}=\|\cdot\|_{D}$, and Theorem 7.2 then shows that the computational solution $\tilde{u}_{M}$ has the same convergence rate as the best approximation $u_{M}^{*}$. 
The computational solution $\tilde{u}_{M}$ is obtained via 7.1 from a stably discretized linear system, and we assume that we perform inexact minimization of $\left\|R_{N}\left(u^{*}-u_{M}\right)\right\|_{V_{N}}$.

In the strong case, this is best linear discrete Chebyshev approximation on sufficiently many points, i.e. a linear optimization problem. In the univariate case with $U_{M}$ being a trial space of polynomials of degree $M-1$ on an interval $I$, a discretization on $N \geq M$ test points forming a set $P_{N}$ will always have a stability inequality

$$
\|u\|_{\infty, I} \leq C(M, N)\|u\|_{\infty, P_{N}}
$$

of the form (5.2), but the stability constant varies. For $M=N$ equidistant points, the constant $C(M, M)$ grows exponentially with $M$, and for Chebyshev-distributed test points it still grows like $\log M$. Uniform stability holds for $N=\mathscr{O}\left(M^{2}\right)$ equidistant points, as follows from a standard argument going back to the notion of norming sets [15] and using Markov's inequality [33, Ch. 3.3]. Theorem 5.3 only proves existence of a uniformly stable discretization, but this example shows that there may be a considerable amount of oversampling or overtesting behind the scene.

If stability is uniform, nodal bases written in terms of values at $M$ nodes $x_{j}$ will trivially lead to $\left|u\left(x_{j}\right)\right| \leq\|u\|_{\infty, I} \leq C\|u\|_{\infty, P_{N}}$ for all $u \in U_{M}$, which is (12.2).

The weak case discretizes by $N$ well-chosen normalized test functionals $\lambda_{v_{j}}$ with normalized Riesz representers $v_{j} \in L_{2}(\Omega)$, and the quantity $\left\|R_{N}\left(u^{*}-u_{M}\right)\right\|_{V_{N}}$ to be minimized is

$$
\max _{1 \leq j \leq N}\left|\left(v_{j}, u^{*}-u_{M}\right)_{L_{2}(\Omega)}\right| .
$$

Our theory shows that the test functionals can be chosen to render uniform stability, but there is a trivial standard choice via the $M$ functionals represented by an orthonormal basis $v_{1}, \ldots, v_{M}$ of $U_{M}$. Then the above minimization produces the best approximation $u_{M}^{*}$ to $u^{*}$ from $U_{M}$ without any oversampling.

In both cases, Theorem 7.4 is applicable, and we see that we the $L_{\infty}$ or $L_{2}$ convergence rates of the non-discrete best approximations carry over to the discrete approximations.

To compare the difference of convergence rates between weak and strong formulations for a given fixed trial space $U_{M}$, we see immediately that the $L_{2}$ convergence rate is never worse than the $L_{\infty}$ rate, but it is taken in a weaker norm. If users insist on the best possible convergence rate in $L_{2}$, they should take a weak form, at the expense of a sufficiently good numerical integration. But the $L_{\infty}$ error of their solution will clearly not have a better $L_{\infty}$ convergence rate than the strong solution.

Both computational approximations, weak or strong, converge like the best approximations in the respective data norm, and this is a fair deal. Convergences can be spectral in certain cases, e.g. in case of univariate functions on an interval $I$ that have a complex extension that is analytic in a region of the complex plane containing $I$ in its interior. This shows how the theory applies to spectral convergence situations without change. 
But, of course, there is the extreme case where the solution is only in $L_{2}$ and not in $C(\Omega)$. Then the strong technique is undefined. But then the weak technique is forced to have the weak data given directly, without numerical integration, because the latter is as unfeasible as the strong technique.

All other examples will show a very similar behavior, differing only in their data maps.

\subsection{Standard Homogeneous Weak Poisson Problem}

We fix a bounded Lipschitz domain $\Omega \subset \mathbb{R}^{d}$ and consider the weak Dirichlet problem $-\Delta u=f$ with homogeneous boundary conditions. This works on the Hilbert space $U:=H_{0}^{1}(\Omega)$ with the inner product

$$
(u, v)_{1}:=\int_{\Omega} \nabla^{T} u(x) \nabla v(x) d x \text { for all } u, v \in U,
$$

and the standard (global) weak formulation asks for a function $u \in U$ with

$$
(u, v)_{1}=(f, v)_{L_{2}(\Omega)} \text { for all } v \in U=H_{0}^{1}(\Omega) .
$$

In the sense of this paper, the functionals are

$$
\lambda_{v}: u \mapsto \lambda_{v}(u)=(u, v)_{1} \text { for all } u, v \in U
$$

and the problem takes the form 1.1 with

$$
\begin{aligned}
\Lambda & :=\left\{\lambda_{v}: v \in U,\|v\|_{U}=1\right\} \subset U^{*} \\
f_{\lambda_{v}} & =\left(u^{*}, v\right)_{1}=(f, v)_{L_{2}(\Omega)} \text { for all } \lambda_{v} \in \Lambda
\end{aligned}
$$

where $u^{*} \in U$ is the true solution.

To check the well-posedness in the sense of section 2, we get

$$
\|D u\|_{V}=\sup _{\lambda_{v} \in \Lambda}\left|\lambda_{v}(u)\right|=\sup _{v \in U,\|v\|_{U}=1}\left|(u, v)_{U}\right|=\|u\|_{U}=:\|u\|_{W P}
$$

proving well-posedness, and the data map $D$ is an isometry.

We now consider fairly arbitrary trial spaces $U_{M} \subset U=H_{0}^{1}(\Omega)$ to allow standard or extended or generalized finite elements, or even certain spectral methods of Galerkin type. Theorem 7.4 is applicable, and we see that we get the convergence rate of approximations to the true solution in $U=H_{0}^{1}(\Omega)$. This is well-known from finite elements, but it holds in general, provided that MRD testing is done. It applies to Petrov-Galerkin methods and spectral techniques of Galerkin type. The rate mainly depends on the smoothness of the solution and on the trial space chosen.

For the standard finite-element situation with piecewise linear elements, this yields $\mathscr{O}(h)$ convergence in $H_{0}^{1}(\Omega)$, as usual for that regularity. To reach $\mathscr{O}\left(h^{2}\right)$ convergence in $L_{2}(\Omega)$ under $H^{2}(\Omega)$ regularity, the Aubin-Nitsche trick is an add-on that is not covered by our theory. But it follows from the fact that the best approximation to $u^{*}$ in $H_{0}^{1}(\Omega)$ automatically has $\mathscr{O}\left(h^{2}\right)$ convergence in $L_{2}$ under $H^{2}(\Omega)$ regularity. This is independent of PDE solving, it is a property of Approximation Theory. 


\subsection{Collocation Methods}

We now want to focus on the general statement

All linear PDE or ODE problems can be numerically solved by collocation in sufficiently many points in such a way that the convergence rate in the wellposedness norm is at least the rate of the trial space data approximation.

This, of course, includes pseudospectral methods. But we have to add more details to show how it follows from Theorem 7.4. We only have to show that collocation is an MRD discretization and pick a suitable form of well-posedness.

The space $U$ should be a normed linear space of functions on a domain $\Omega$ with boundary $\Gamma$, for instance a Sobolev space. To keep things simple, we assume that the analytic problem is posed in strong form by evaluating a linear elliptic second-order differential operator $L$ on points of the domain and a linear boundary operator $B$ on the boundary, i.e.

$$
\begin{array}{ll}
L u(x)=f(x), & \text { for all } x \in \Omega \\
B u(y)=g(y), & \text { for all } y \in \Gamma
\end{array}
$$

where $f$ and $g$ are given functions on $\Omega$ and $\Gamma$. Introducing continuous functionals $\lambda_{x}(u):=L u(x)=\delta_{x} \circ L$ and $\mu_{y}(u):=B u(y)=\delta_{y} \circ B$ on $U$ one gets a problem of the form 1.1 with

$$
\Lambda:=\left\{\lambda_{x}: x \in \Omega\right\} \cup\left\{\mu_{y}: y \in \Gamma\right\}
$$

and it should be clear that one can allow more than two operators, and combinations of different boundary conditions.

From here there are different ways to proceed towards well-posedness, but we can ignore well-posedness for a moment. We normalize all functionals as elements of $U^{*}$ and pose the problem in the form 1.1 with $f_{\lambda}:=\lambda\left(u^{*}\right)$ for all $\lambda \in \Lambda$. Then $|\lambda(u)| \leq\|u\|_{U}$ for all $u \in U, \lambda \in \Lambda$, and there is no problem to define the space $V$ and the restrictions via taking suprema. We can apply Theorems 5.3 and 7.2 without assuming well-posedness, and we see that we can work on any trial space $U_{M}$, but all results only hold in the data norm. Comparing with any existing good approximation $u_{M}^{*}$ to $u^{*}$ from $U_{M}$, we get some $\tilde{u}_{M} \in U_{M}$ by a discrete computational method such that

$$
\sup _{\lambda \in \Lambda}\left|\lambda\left(\tilde{u}_{M}-u^{*}\right)\right| \leq\left(2 C_{A}+3\right) \sup _{\lambda \in \Lambda}\left|\lambda\left(u_{M}^{*}-u^{*}\right)\right| \leq\left(2 C_{A}+3\right)\left\|u_{M}^{*}-u^{*}\right\|_{U}
$$

due to normalization of the functionals, and if we use some $u_{M}^{*}$ with small $\left\|u_{M}^{*}-u^{*}\right\|_{U}$, we get the above statement. The backward error analysis of Section 8 will be applicable here.

In the above setting, the most natural well-posedness condition would be of the form

$$
\|u\|_{W P} \leq C \max \left(\|L u\|_{\infty, \Omega},\|B u\|_{\infty, \Gamma}\right)
$$

for a suitable norm $\|.\|_{W P}$ on $U$. This holds for $U:=C^{2}(\Omega) \cap C(\bar{\Omega})$ with the sup norm in $U$ [8, (2.3), p. 14] for uniformly elliptic operators $L$ and Dirichlet boundary data. 
This implies by Theorem 7.4 that for such problems the convergence rate of the Trial Space Data Approximation carries over to the convergence rate of collocation in the sup norm.

As a special example we consider unsymmetric collocation [17] by translates of the kernel $K$ of a Hilbert space $U$, applied to a Dirichlet problem of the form (13.1) on a domain $\Omega \subset \mathbb{R}^{d}$. The trial space $U_{M}$ is spanned by kernel translates $v_{j}:=K\left(\cdot, x_{j}\right), 1 \leq$ $j \leq M$ for nodes $x_{1}, \ldots, x_{M} \in \Omega$, but this is not a stable basis. A nodal basis in the sense of Section 12 consists of the Lagrange basis $u_{1}, \ldots, u_{M}$ spanning the same trial space. Collocation is done via the functionals defined for (13.2), and to make them continuous we can take a space like $U:=H^{m}(\Omega)$ with some $m>2+d / 2$. We have well-posedness in the sense of (13.3) in the sup-norm.

Theorem 13.4. Unsymmetric collocation in the sense of E. Kansa [17] has the property that for each possible trial space spanned by kernel translates there is a selection of test functionals such that the stiffness matrix, when written in terms of the nodal basis, has a uniform stability property (12.2). If solved by residual minimization along the lines of Section 7 error bounds follow from Corollary 7.3 or (12.3). Convergence rates in the sup norm are obtained from the rate of convergence of second derivatives in the sup norm of interpolants of the true solution by the trial space.

This provides many explicit convergence rates via standard results on interpolation by translates of kernels [33, Chapter 11]. For instance, the convergence for the WhittleMatérn kernel reproducing $H^{m}\left(\mathbb{R}^{d}\right)$ for $m>2+d / 2$ is like $\mathscr{O}\left(h^{m-2-d / 2}\right)$ in terms of the fill distance $h:=\sup _{y \in \Omega} \min _{1 \leq j \leq M}\|y-x\|_{2}$, while the convergence is exponential for kernels like the Gaussian or multiquadrics.

The functionals in (13.2) are a mixture of two kinds, but Theorem 5.3 and Lemma 6.1 do not say how to achieve a uniformly stable balance between testing $B$ on the boundary and testing $L$ in the interior. Future work should address this problem, and Section 13.1 suggests that there might be quite some overtesting needed for uniform stability. Square collocation systems can even be singular [13], such that overtesting is necessary in general.

All of this readily generalizes to plenty of other linear well-posed PDE problems, and readers can use the tools of this paper to assemble what they need. Note that unsymmetric collocation is a pseudospectral method in the sense of the literature (see e.g. [11, 12, 9]) on spectral methods, and this paper provides a general way to assess convergence of pseudospectral methods. Since we write the analytic and the computational problems in terms of arbitrary functionals, this approach also covers spectral methods in Tau form.

\subsection{Weak Dirichlet Problems}

The standard finite element procedures for solving Dirichlet problems for the Laplace operator on bounded domains $\Omega \subset \mathbb{R}^{d}$ use strong data on the boundary and weak data 
in the interior. The data functionals are

$$
\begin{aligned}
\Lambda_{1} & :=\left\{\lambda_{v}: u \mapsto(\nabla u, \nabla v)_{L_{2}(\Omega)} \text { for all } v \in H_{0}^{1}(\Omega),\|\nabla v\|_{L_{2}(\Omega)}=1\right\}, \\
\Lambda_{2} & :=\left\{\delta_{y}: y \in \Gamma:=\partial \Omega\right\}, \\
\Lambda & :=\Lambda_{1} \cup \Lambda_{2} .
\end{aligned}
$$

This leads to the data norm

$$
\|u\|_{D}=\max \left(\|u\|_{\infty, \Gamma},\|\nabla u\|_{L_{2}(\Omega)}\right)
$$

if we take the sup over all functionals as in Section 4 It is well-defined on the space $U:=H^{1}(\Omega) \cap C(\bar{\Omega})$. Using the Poincaré inequality and the Maximum Principle [16] after splitting $u$ into a harmonic part with boundary conditions and a function in $H_{0}^{1}(\Omega)$ satisfying the differential equation, we get a well-posedness inequality

$$
\|u\|_{L_{2}(\Omega)} \leq C\|u\|_{D}=C \max \left(\|u\|_{\infty, \Gamma},\|\nabla u\|_{L_{2}(\Omega)}\right) \text { for all } u \in U .
$$

Note that the Sobolev inequality forbids to use the sup norm on the left-hand side for space dimension $d>1$.

Whatever the chosen trial spaces in $U$ are, Theorem 7.4 shows that the convergence rate in $L_{2}$ of uniformly stabilized computational methods will be the convergence rate of the Trial Space Data Approximation, i.e. with respect to $\|u\|_{\infty, \Gamma}$ and $\|\nabla u\|_{L_{2}(\Omega)}$. If the trial space is spanned by translates of the Whittle-Matérn kernel reproducing $H^{m}\left(\mathbb{R}^{d}\right)$ for $m>1+d / 2$, the rate is $\mathscr{O}\left(h^{m-1-d / 2}\right)$ in terms of the fill distance of the trial nodes [33].

For standard finite elements, the above approach yields $\mathscr{O}(h)$ convergence in $L_{2}$. This is without the Aubin-Nitsche trick, and it does not use $H^{2}$ regularity.

The Aubin-Nitsche trick has nothing to do with finite elements and weak problems. It is a feature of Approximation Theory, doubling a convergence rate for certain nested approximations in Hilbert spaces under additional regularity assumptions. This is wellknown from splines [1, 5.10] and kernel-based methods [23]. In the context of this paper, one considers the best approximation to the true solution in $H_{0}^{1}(\Omega)$, and it will automatically yield $\mathscr{O}\left(h^{2}\right)$ convergence under $H^{2}$ regularity, but only for zero boundary conditions.

\subsection{Weak-Strong Comparison}

If we compare with what we had in the strong case, the situation for fixed trial spaces is roughly as follows:

1. The weak case has $L_{2}$ convergence at the convergence rate for first derivatives,

2. the strong case has $L_{\infty}$ convergence at the convergence rate for second derivatives. 
This usually yields a slightly better rate for the weak case, as we saw when comparing $\mathscr{O}\left(h^{m-1-d / 2}\right)$ with $\mathscr{O}\left(h^{m-2-d / 2}\right)$ for the trial space spanned by translates of the Whittle-Matérn kernel. On the downside, weak methods usually have to perform numerical integration at an accuracy that complies with the convergence rate, and they converge in a weaker norm.

If one fixes the available finite data and then looks for an error-optimal solution in a fixed Reproducing Kernel Hilbert Space, the above discussion about differences between strong and weak methods becomes obsolete. The optimal solution is always the one described in Section 10, and it is furnished by symmetric collocation [26]. Since it allows arbitrary evaluation functionals $\mu$ in Theorem 10.1, it is pointwise and $L_{\infty}$-optimal by taking functionals $\mu=\delta_{x}$, and $L_{2}$-optimal by taking all functionals $\mu=\lambda_{v}=(., v)_{L_{2}(\Omega)}$.

\subsection{MLPG}

We stay with the Dirichlet problem for the Laplacian, for simplicity, and describe the standard variation of the Meshless Local Petrov-Galerkin [5, 4] method. The difference to the standard weak formulation is that the integrals are localized and the boundary integrals are kept. This means that on small subdomains $\Omega_{h} \subset \Omega$ with boundaries $\Gamma_{h} \subset \bar{\Omega}$ the strong equation $-\Delta u=f$ is integrated against a test function $v_{h}$ to define functionals of the form

$$
u \mapsto \lambda_{\Omega_{h}, v_{h}}(u)=\frac{-1}{\operatorname{vol}\left(\Omega_{h}\right)} \int_{\Omega_{h}} v_{h} \cdot \Delta u
$$

that are continuous on $U:=C^{2}(\Omega) \cap C(\bar{\Omega})$, and the problem (13.1) takes the form 1.11 via

$$
\begin{aligned}
\lambda_{\Omega_{h}, v_{h}}(u) & =\frac{1}{\operatorname{vol}\left(\Omega_{h}\right)} \int_{\Omega_{h}} v_{h} \cdot f & & \text { for all } \Omega_{h} \subset \Omega, v_{h} \in C\left(\Omega_{h}\right) \\
\delta_{y}(u) & =g(y) & & \text { for all } y \in \Gamma
\end{aligned}
$$

for given continuous functions $f$ on $\Omega$ and $g$ on $\Gamma$. One can restrict the domains $\Omega_{h}$ and the test functions $v_{h}$ further, and allow other ways of handling the boundary conditions. Furthermore, the above functionals are usually transformed by integration by parts before they are implemented, but we deal with this later.

The goal is to prove some form of well-posedness for the analytic problem, and this seems to be missing completely in the rich literature on the MLPG method. On the space $U=C^{2}(\Omega) \cap C(\bar{\Omega})$ we know that $\left[13.3\right.$ holds for $\|\cdot\|_{W P}=\|\cdot\|_{\infty, \bar{\Omega}}$, and we assert

$$
\|u\|_{\infty, \bar{\Omega}} \leq C\left(\sup _{\Omega_{h}, v_{h}}\left|\lambda_{\Omega_{h}, v_{h}}(u)\right|+\|u\|_{\infty, \Gamma}\right) \text { for all } u \in U .
$$

But this follows from (13.3) by setting $f:=\Delta u$ in 
Lemma 13.7. For each continuous function $f$ on some compact domain $\Omega \subset \mathbb{R}^{d}$ the norms

$$
\|f\|_{\infty, \Omega}=\|f\|_{I}:=\sup _{\Omega_{h} \subset \Omega} \frac{1}{\operatorname{vol}\left(\Omega_{h}\right)}\left|\int_{\Omega_{h}} f\right|
$$

coincide, where the diameter of the admissible sets $\Omega_{h}$ can be bounded above by some arbitrary $r>0$, if required. One can also restrict the subdomains $\Omega_{h}$ to balls or cubes intersected with $\Omega$.

Proof: Clearly $\|f\|_{I} \leq\|f\|_{\infty, \Omega}$ holds. To prove $\|f\|_{I} \geq\|f\|_{\infty, \Omega}$, assume $f \neq 0$ with $\|f\|_{\infty, \Omega}=f(\tilde{x})>0$ for some $\tilde{x} \in \Omega$. Then pick an arbitrary $\varepsilon<f(\tilde{x}) / 2$ and an arbitrary $r>0$ and choose $\Omega_{h}$ to be a subdomain of the set of points $x \in \Omega$ with

$$
0<(1-\varepsilon) f(\tilde{x}) \leq f(x) \leq f(\tilde{x}),\|x-\tilde{x}\|_{2} \leq r .
$$

For instance, one can take the intersection of sufficiently small balls or cubes around $\tilde{x}$ with the domain $\Omega$, or if $\tilde{x}$ is on the boundary, one may move slightly into the interior and ensure $\Omega_{h}$ to be in the interior of the domain. Then

$$
(1-\varepsilon)\|f\|_{\infty, \Omega}=(1-\varepsilon) f(\tilde{x}) \leq \frac{1}{\operatorname{vol}\left(\Omega_{h}\right)} \int_{\Omega_{h}} f \leq f(\tilde{x})=\|f\|_{\infty, \Omega} .
$$

Note that this proves well-posedness only on $U=C^{2}(\Omega) \cap C(\bar{\Omega})$, not on a larger space, but for all possible test functions and domain shapes and sizes. The boundary conditions can be rephrased by weak functionals taking means, using Lemma 13.7 again, now setting $f:=g$ and working on the boundary.

Any a-priori renormalization of all available functionals will possibly spoil this argument. But as soon as finitely many functionals are selected for computation, one can renormalize for the computational procedure.

If integration by parts is applied to the functionals, they change their computational form without changing their value, and this is used in the known variations of the MLPG technique. For instance,

$$
\begin{aligned}
\lambda_{\Omega_{h}, v_{h}}(u) & =\frac{1}{\operatorname{vol}\left(\Omega_{h}\right)} \int_{\Omega_{h}} \nabla^{T} v_{h} \cdot \nabla u-\frac{1}{\operatorname{vol}\left(\Omega_{h}\right)} \int_{\Gamma_{h}} v_{h} \frac{\partial u}{\partial n} \\
& =\frac{-1}{\operatorname{vol}\left(\Omega_{h}\right)} \int_{\Omega_{h}} \Delta v_{h} \cdot u+\frac{1}{\operatorname{vol}\left(\Omega_{h}\right)} \int_{\Gamma_{h}} u \frac{\partial v_{h}}{\partial n}-\frac{1}{\operatorname{vol}\left(\Omega_{h}\right)} \int_{\Gamma_{h}} v_{h} \frac{\partial u}{\partial n}
\end{aligned}
$$

are two ways to rewrite the functionals on different domains with different admissible test functions. The basic well-posedness on $U=C^{2}(\Omega) \cap C(\bar{\Omega})$ will stay as is, because the sup of all these functionals will be bounded above by $\|\Delta u\|_{\infty, \Omega}$, as long as there are no other upper bounds proven.

The method called MLPG5 uses constant test functions like in Lemma13.7 Then the functionals take the extremely simple form

$$
\lambda_{\Omega_{h}, v_{h}}(u)=-\frac{1}{\operatorname{vol}\left(\Omega_{h}\right)} \int_{\Gamma_{h}} \frac{\partial u}{\partial n},
$$


i.e. they are only integrals of the normal derivative on subdomain boundaries. Nevertheless, Lemma 13.7 holds, and there is well-posedness in the sup norm on $U=$ $C^{2}(\Omega) \cap C(\bar{\Omega})$. It is an open problem to prove other well-posedness inequalities after fixing a special form of the functionals. The above technique via Lemma13.7 always goes back to 13.3), whatever the form of the functionals is after integration by parts. Therefore the convergence theory for given trial spaces will be the same as for the strong collocation methods in Section 13.3 .

Theorem 13.8. If the Meshless Local Petrov-Galerkin method is carried out

1. for a well-posed second-order elliptic problem,

2. using sufficiently many well-chosen test functionals (13.5) along the lines of Theorem 5.3

3. and applying a residual minimization algorithm as in Section 7 for solving the overdetermined system approximatively,

the algorithm is convergent with uniform stability, and the convergence rate in the sup norm is the rate of the Trial Space Data Approximation. This rate is at least as good as for strong collocation using the same trial spaces.

Depending on the PDE problem, the smoothness of the true solution, and the trial space chosen, this yields various convergence results, up to spectral convergence. In most applications, the trial functions are shape functions provided by Moving Least Squares, and raising the degree of the local polynomials will increase the convergence rate appropriately [18, 32, 2, 3]. Readers are encouraged to apply the framework of this paper to derive special convergence results for various trial spaces and different variations of the MLPG technique. In particular, an extension to elasticity problems should be quite useful.

But the methods of this paper always assume the functionals to be given exactly, not approximately. Only their values can be noisy, as in Section 8 This excludes various interesting applications, namely the Direct Meshless Local Petrov Galerkin (DMLPG) technique [20] and localized kernel-based methods that provide sparse stiffmess matrices [30, 22, 31, 29, 34].

\section{References}

[1] J.H. Ahlberg, E.N. Nilson, and J.L. Walsh. The theory of splines and their applications, volume 38 of Mathemtics in science and engineering. Academic Press, 1967.

[2] M.G. Armentano. Error estimates in Sobolev spaces for moving least square approximations. SIAM J. Numer. Anal., 39(1):38-51, 2001.

[3] M.G. Armentano and R.G. Durán. Error estimates for moving least square approximations. Appl. Numer. Math., 37:397-416, 2001. 
[4] S. N. Atluri. The meshless method (MLPG) for domain and BIE discretizations. Tech Science Press, Encino, CA, 2005.

[5] S. N. Atluri and T.-L. Zhu. A new meshless local Petrov-Galerkin (MLPG) approach in Computational Mechanics. Computational Mechanics, 22:117-127, 1998.

[6] T. Belytschko, Y. Krongauz, D.J. Organ, M. Fleming, and P. Krysl. Meshless methods: an overview and recent developments. Computer Methods in Applied Mechanics and Engineering, special issue, 139:3-47, 1996.

[7] K. Böhmer and R. Schaback. A nonlinear discretization theory. Journal of Computational and Applied Mathematics, 254:204-219, 2013.

[8] D. Braess. Finite Elements. Theory, Fast Solvers and Applications in Solid Mechanics. Cambridge University Press, 2001. Second edition.

[9] C. Canuto, M. Y. Hussaini, A. Quarteroni, and T. A. Zang. Spectral methods. Scientific Computation. Springer, Berlin, 2007. Evolution to complex geometries and applications to fluid dynamics.

[10] O. Davydov and R. Schaback. Error bounds for kernel-based numerical differentiation. Draft, 2013.

[11] B. Fornberg and D.M. Sloan. A review of pseudospectral methods for solving partial differential equations. Acta Numerica, pages 203-267, 1994.

[12] Bengt Fornberg. A practical guide to pseudospectral methods, volume 1 of Cambridge Monographs on Applied and Computational Mathematics. Cambridge University Press, Cambridge, 1996.

[13] Y. C. Hon and R. Schaback. On unsymmetric collocation by radial basis functions. Appl. Math. Comput., 119(2-3):177-186, 2001.

[14] Y. C. Hon, R. Schaback, and X. Zhou. An adaptive greedy algorithm for solving large RBF collocation problems. Numer. Algorithms, 32(1):13-25, 2003.

[15] K. Jetter, J. Stöckler, and J.D. Ward. Error estimates for scattered data interpolation on spheres. Mathematics of Computation, 68:733-747, 1999.

[16] J. Jost. Partial differential equations, volume 214 of Graduate Texts in Mathematics. Springer-Verlag, New York, 2002. Translated and revised from the 1998 German original by the author.

[17] E. J. Kansa. Application of Hardy's multiquadric interpolation to hydrodynamics. In Proc. 1986 Simul. Conf., Vol. 4, pages 111-117, 1986.

[18] D. Levin. The approximation power of moving least-squares. Mathematics of Computation, 67:1517-1531, 1998. 
[19] L. Ling and R. Schaback. On adaptive unsymmetric meshless collocation. In S.N. Atluri and A.J.B. Tadeu, editors, Proceedings of the 2004 International Conference on Computational \& Experimental Engineering and Sciences, Advances in Computational \& Experimental Engineering \& Sciences. Tech Science Press, 2004. paper \# 270.

[20] D. Mirzaei and R. Schaback. Direct Meshless Local Petrov-Galerkin (DMLPG) method: A generalized MLS approximation. Applied Numerical Mathematics, http://dx.doi.org/10.1016/j.apnum.2013.01.002, 2013.

[21] C. Rieger, B. Zwicknagl, and R. Schaback. Sampling and stability. In M. Dæhlen, M.S. Floater, T. Lyche, J.-L. Merrien, K. Mørken, and L.L. Schumaker, editors, Mathematical Methods for Curves and Surfaces, volume 5862 of Lecture Notes in Computer Science, pages 347-369, 2010.

[22] B. Šarler. From global to local radial basis function collocation method for transport phenomena. In Advances in meshfree techniques, volume 5 of Comput. Methods Appl. Sci., pages 257-282. Springer, Dordrecht, 2007.

[23] R. Schaback. Improved error bounds for scattered data interpolation by radial basis functions. Mathematics of Computation, 68:201-216, 1999.

[24] R. Schaback. Convergence of unsymmetric kernel-based meshless collocation methods. SIAM J. Numer. Anal., 45(1):333-351 (electronic), 2007.

[25] R. Schaback. Unsymmetric meshless methods for operator equations. Numer. Math., 114:629-651, 2010.

[26] R. Schaback. A computational tool for comparing all linear PDE solvers. to appear in Advances of Computational Mathematics, http://www.num.math.uni-goettingen.de/schaback/research/group.html, 2013.

[27] R. Schaback. Greedy sparse linear approximations of functionals from nodal data. to appear in Numerical Algorithms, DOI: 10.1007/s11075-013-9806-8, 2014.

[28] R. Schaback and H. Wendland. Adaptive greedy techniques for approximate solution of large RBF systems. Numer. Algorithms, 24(3):239-254, 2000.

[29] Q. Shen. Local RBF-based differential quadrature collocation method for the boundary layer problems. Eng. Anal. Bound. Elem., 34(3):213-228, 2010.

[30] C. Shu, H. Ding, and K. S. Yeo. Computation of incompressible Navier-Stokes equations by local RBF-based differential quadrature method. CMES Comput. Model. Eng. Sci., 7(2):195-205, 2005.

[31] D. Stevens, H. Power, M. Lees, and H. Morvan. The use of PDE centres in the local RBF Hermitian method for 3D convective-diffusion problems. J. Comput. Phys., 228(12):4606-4624, 2009.

[32] H. Wendland. Local polynomial reproduction and moving least squares approximation. IMA Journal of Numerical Analysis, 21:285-300, 2001. 
[33] H. Wendland. Scattered Data Approximation. Cambridge University Press, 2005.

[34] G.M. Yao, Siraj ul Islam, and B. Šarler. Assessment of global and local meshless methods based on collocation with radial basis functions for parabolic partial differential equations in three dimensions. Eng. Anal. Bound. Elem., 36(11):1640$1648,2012$. 